\title{
NOTA
}

\section{La idea de justicia en Amartya Sen y la educación jurídica en las facultades jesuitas}

César Arjona' y Josep F. Mària, S. I. ${ }^{2}$

Resumen: Esta nota parte de las críticas que Amartya Sen realiza sobre las teorías de la justicia dominantes en la actualidad y los problemas detectados en el modelo de educación jurídica dominante. A partir de la relación existente entre ambos aspectos, se exponen las orientaciones que Sen propone para superar los problemas detectados, las cuales, en opinión de los autores, están conectadas estrechamente con la promoción de la justicia que la Compañía de Jesús ha adoptado como principio integrador de su misión. El texto finaliza analizando cómo esa misión puede plasmarse en una facultad de Derecho jesuita.

Palabras clave: teoría de la justicia, educación jurídica, promoción de la justicia.

Fecha de recepción: 4 de julio de $20 \mathrm{II}$.

Fecha de admisión definitiva: 16 de febrero de 2012 .

\footnotetext{
' Departamento de Derecho público, ESADE (Universidad Ramon Llull), Barcelona.

2 Departamento de Ciencias sociales, ESADE (Universidad Ramon Llull), Barcelona.
} 


\section{Amartya Sen's concept of justice and legal education in Jesuit law schools}

Abstract: This article refers to the criticism made by Amartya Sen about the predominant theories regarding justice today and the problems detected in the principal model of law training. Starting with the relationship between these two aspects, there is a presentation of the guidelines proposed by Sen to overcome the problems identified, which the authors feel are closely connected to that promotion of justice which the Society of Jesus has adopted as the basic integrating premise of its mission. The text ends with an analysis of how this mission can be effectuated in a Jesuit law school.

Key words: theory of justice, law school, promotion of justice.

\section{L'idée de justice chez Amatya Sen ef I'éducation juridique dans les facultés jésuites}

Résumé: Nous partons, dans cette note, des critiques qu'Amartya Sen réalise sur les théories dominantes de la justice actuellement et des problèmes détectés dans le modèle d'éducation juridique dominant. À partir des relations existantes entre ces deux aspects, nous exposons les orientations que Sen propose pour surmonter les problèmes détectés, lesquelles, selon les auteurs, sont en étroite relation avec la promotion de la justice que la Compagnie de Jésus a adoptée comme principe intégrateur de sa mission. Le texte finalise en analysant comment cette mission peut être mise en place dans une faculté de Droit Jésuite.

Mots clé: théorie de la justice, éducation juridique, promotion de la justice.

\section{Introducción, y planteamiento del problema}

En su última obra, titulada The Idea of Justice, el premio Nobel de Economía de origen indio Amartya Sen pone en cuestión las teorías de la justicia dominantes, destacando su inhabilidad para percibir en su verdadera dimensión la naturaleza de los muchos y muy graves problemas de injusticia global, y en consecuencia para colaborar en la lucha contra los mismos ${ }^{3}$.

Estos problemas tienen mucho que ver con lo que el decreto 3 de la Congregación general $34^{a}$ denomina las "nuevas dimensiones de la justicia", título bajo el cual se enumeran los distintos ámbitos en los que está en juego la promoción de

\footnotetext{
${ }^{3}$ A. SEN (2009) The Idea of Justice, Londres, Allen Lane, Penguin, 28+468 pp. (hay traducción caste-
} llana: La idea de justicia, Madrid 2010, Taurus, 499 pp.) 
la justicia que la Compañía de Jesús ha adoptado como principio integrador de su misión (nn. 5-10). Entre ellos se encuentran los riesgos y las oportunidades implicados en la globalización (n. 7), que son destacados específicamente en la siguiente Congregación general $35^{\mathrm{a}}$, decreto $3, \mathrm{nn} .8-11$, donde se habla de un nuevo contexto para la misión jesuita, el contexto del mundo global, repleto de tensiones y paradojas (n. 11).

A los autores de este comentario, profesores ambos que imparten, en una facultad de Derecho, asignaturas que tratan sobre el concepto de justicia, les preocupa el hecho de que el modelo de educación jurídica dominante (a escala global, aunque con significativos matices) margina en gran medida la cuestión de la justicia, apartándola del núcleo duro de la pedagogía jurídica. En el estudio de la obra de Sen, que utiliza en su teoría de la justicia perspectivas provenientes de muchos ámbitos del conocimiento, entre ellos la jurisprudencia o pensamiento jurídico, hemos encontrado indicaciones claras del porqué de esa marginación. Eso es posible dado que existe una correspondencia entre las teorías de la justicia dominantes que Sen critica y el modelo de educación jurídica también dominante al que nos referimos.

En la sección segunda de este trabajo destacaremos algunas de las críticas de Sen a la teoría de la justicia dominante, que él identifica con la teoría contractualista de John Rawls, y trasladaremos esos puntos críticos al ámbito de la educación jurídica. Apuntaremos las orientaciones que Sen propone para superarlos, que, como trataremos de reflejar en la sección tercera, pueden relacionarse estrechamente con la misión de la Compañía de Jesús. En la última sección nos referiremos a cómo esa misión puede plasmarse en una facultad de Derecho jesuita.

\section{La obra de Sen The Idea of Justice}

Sen construye su obra como una crítica general a la teoría de la justicia dominante en el pensamiento contemporáneo, que es la del norteamericano John Rawls. Sen reconoce el mérito de Rawls, a quien considera la culminación de la tradición contractualista que inauguró Hobbes y prosiguieron filósofos como Locke o Rousseau. A pesar de ello, Sen critica una serie de elementos esenciales de la teoría de Rawls que ponen en cuestión la capacidad de esta teoría de enfrentarse a las situaciones de injusticia del mundo contemporáneo.

Dentro de esos diversos elementos, vamos a destacar aquí dos que son los que de manera más clara se corresponden con características muy arraigadas en la 
pedagogía jurídica contemporánea. A nuestro entender, dichos elementos sirven para explicar la relativa marginación de las cuestiones de justicia en las facultades de Derecho.

Esos dos elementos son el carácter (a) institucionalista y (b) excluyente de la teoría de la justicia de Rawls.

\section{I. El carácter institucionalista de la teoría dominante}

Al hablar del carácter institucionalista, Sen se refiere a que en la teoría de Rawls la justicia equivale a un conjunto de instituciones justas, quedando relativamente fuera del campo de atención las vidas humanas reales, los comportamientos de la gente, y lo que Sen a menudo denomina realizaciones sociales.

La idea de que la justicia se alcanza mediante el diseño y la implementación adecuadas de ciertas instituciones es, según Sen, gravemente parcial, al despreciar elementos tanto o más importantes en la consideración de la justicia, como son los comportamientos reales de los individuos y las consecuencias sociales que se producen. Creer que la clave de la justicia se halla en la acción espontánea de la gente puede ser ingenuo, pero un enfoque exclusivamente institucional no bastará para atacar las causas reales de la injusticia. Por tanto, la consideración de la justicia debe implicar un equilibrio entre instituciones y realizaciones, y si de lo que se trata es de mejorar la sociedad, habrá que tener en cuenta que existe una mutua dependencia entre la reforma institucional y los cambios en el comportamiento social. Por eso afirma Sen que las instituciones no representan la justicia por ellas mismas. Sí es cierto que son útiles para promover la justicia, pero que realmente lo logren es algo que depende de otras circunstancias del comportamiento humano, circunstancias que las teorías contractualistas han despreciado considerablemente ${ }^{4}$.

En este punto, Sen lleva la argumentación al terreno jurídico al ilustrar el problema del institucionalismo mediante una distinción que toma de la jurisprudencia india clásica: la distinción entre niti y nyaya. Traducidas del sánscrito clásico, del

${ }^{4}$ Tenemos que buscar instituciones que promuevan la justicia, en lugar de tratar a las instituciones como manifestaciones directas de la justicia, lo cual reflejaría un cierto fundamentalismo institucional (...) Por supuesto, las instituciones cuentan como parte sensible de las realizaciones que se producen a través de ellas, pero difícilmente podemos concentrarnos tan sólo en ellas, ya que las vidas de las personas también están implicadas (cfr. A. SEN, op. cit., pp. 112-113). 
que provienen estas dos palabras, y siempre según Sen, ambas quieren decir justicia. Sin embargo, se refieren a la justicia en dos dimensiones distintas. El niti hace referencia a la organizational propriety and behavioural correctness, mientras que el nyaya apunta hacia un comprehensive concept of realized justice y tiene por tanto en cuenta las consecuencias que se producen en el mundo. Una perspectiva niti se concentra en reglas e instituciones, pero si queremos conocer el mundo que realmente tenemos, y las injusticias que en él suceden, debemos complementar aquella con la perspectiva del nyaya ${ }^{5}$.

En el ámbito de la educación jurídica, este enfoque institucionalista se refleja en una concentración casi exclusiva en la dimensión dogmática del Derecho, esto es, en una dimensión niti. El objetivo pedagógico se entiende principalmente como adquisición de conocimiento, a menudo memorístico, del texto de leyes escritas, y secundariamente de comentarios doctrinales o jurisprudenciales sobre esas leyes y de la forma de las instituciones (tribunales, órganos administrativos, etc) que esas leyes crean. El déficit de nyaya en las facultades de Derecho es patente, y creemos que responsable de un cierto grado de deshumanización de los estudios jurídicos, en tanto estos giran en torno al dominio relativamente acrítico de un conjunto de reglas escritas, por encima de los comportamientos humanos implicados en esas reglas. Contra eso, y siguiendo a Sen, a quien le preocupe la justicia debiera hacer esfuerzos por adoptar enfoques nyaya, dado que los enfoques niti se encuentran necesariamente limitados a una perspectiva institucional que en el mejor de los casos sólo puede captar indirectamente las injusticias del mundo. Si la injusticia arde, quizás desde el niti se pueda sentir el calor más o menos lejano de ese fuego, pero para quemarse es necesario introducirse en el más incómodo mundo del nyaya. Sen es una prueba de su posición al permitir que en su teoría de la justicia trasluzca la motivación personal que le ha llevado a escribirla: la constatación difícilmente soportable de la injusticia real en el mundo.

Uno de los temas secundarios del libro de Sen que aquí tomamos como base de nuestra reflexión, es destacar el origen muy antiguo y no occidental de ideas que en occidente se consideran originales y modernas. Así, por ejemplo, en la contraposición entre niti y nyaya resuena una de las distinciones conceptuales más fértiles en la jurisprudencia occidental contemporánea, principalmente anglo-sajona: la que contrasta el Derecho en los libros [Law in Books] con el Derecho en acción

\footnotetext{
${ }^{5}$ En esta línea, las funciones de las instituciones, las reglas y la organización, a pesar de su importancia, han de ser evaluadas en la perspectiva más amplia e inclusiva de nyaya, que está ligada de manera ineludible al mundo que realmente surge y no sólo a las instituciones o reglas que tenemos por casualidad (cfr. A. SEN, op. cit., pp. 51-52).
} 
[Law in Action]. Según esta distinción, que fue formulada inicialmente por el jurista norteamericano Roscoe Pound hace aproximadamente un siglo, mediante el Derecho en los libros se hace referencia a las reglas escritas que pretenden gobernar las relaciones humanas, mientras que el concepto de Derecho en acción se refiere a las normas que de hecho rigen esas relaciones ${ }^{6}$. La aproximación del Derecho en acción nos obliga a dejar de lado los libros por un momento y acudir a los hechos sociales para conocer la realidad a la que aquellas reglas escritas pretenden aplicarse y las consecuencias reales que produce dicha aplicación. Es, en el sentido que Sen introduce, una aproximación nyaya.

Esta distinción la formuló Pound desde el rechazo hacia una manera de aproximarse al Derecho demasiado apegada a los libros de leyes y aislada de la vida real y del resto de ámbitos del conocimiento científico, social y humanístico. Ese rechazo fue compartido por muchos otros juristas, y el Law in Books frente al Law in Action fue adoptado como lema por la escuela del realismo jurídico, que tuvo una importancia fundamental en la cultura jurídica de Estados Unidos a lo largo del siglo XX. El énfasis en el Derecho en acción se reflejó en características distintivas de la educación jurídica de aquel país, como la utilización extendida del método del caso, la introducción de la educación clínica (clinical legal education) y las simulaciones procesales (moot courts). Elementos de este tipo son sin embargo menos habituales en otros sistemas, y particularmente extraños en los contextos no anglo-sajones ${ }^{7}$.

Todo esto no quiere decir que la adopción de un enfoque nyaya garantice una mayor sensibilidad hacia las cuestiones de la justicia. Sin embargo, aunque no sea suficiente, sí es necesario abrir esa puerta si se quiere trabajar sobre la justicia. Las injusticias tienen más que ver con las vidas humanas y las realizaciones sociales que con las reglas escritas en libros (aunque estas pueden contribuir decisivamente a mantener, agravar o combatir aquellas). Puede que un enfoque niti, que se concentre exclusivamente en el Derecho en los libros, baste para formar técnicos acríticos de la ley, pero eso es algo que no debería constituir el ideal pedagógico de una facultad de Derecho jesuita que desea inspirarse en la idea de justicia tal como ha sido reflejada en las últimas Congregaciones generales.

\footnotetext{
${ }^{6}$ R. Pound (1910) "Law in Books and Law in Action", American Law Review 44, p. 12.
}

7 Un reciente artículo describe la paulatina expansión de la educación clínica por todo el mundo y los beneficios que comporta, aunque destaca también las resistencias frente a este movimiento que son particularmente intensas en el ámbito de la Europa occidental. Ver R. WILSON (2009) "Western Europe: Last Holdout in the Worldwide Acceptance of Clinical Legal Education", German Law Journal 10, p. 823 


\subsection{El carácter excluyente de la teoría dominante}

Cuando Sen critica la teoría de Rawls por excluyente, hace referencia al hecho de que los enfoques contractualistas están por definición limitados a una comunidad política determinada. El pacto social, tanto en la versión rawlsiana como en las más clásicas, se firma entre unos individuos determinados (con exclusión de otros) entre los que se llega a un acuerdo que les vincula a ellos (y no a otros) para establecer una sociedad justa. Esto es coherente con el contexto de la tradición contractualista, que desde su origen en Hobbes tiene por finalidad la legitimación del poder político de un Estado.

Sin embargo, como considera acertadamente Sen, resulta muy limitado valorar la justicia en un país o Estado determinado sin tener en cuenta perspectivas que vienen de fuera de esa comunidad. Eso es así por lo menos por dos razones:

- por un lado, porque en el mundo global en el que de hecho vivimos lo que sucede en un país suele tener efectos importantes en otros países (por ejemplo, sería inaceptablemente limitado hablar sobre la justicia en Iraq sin considerar los procesos que llevaron en Estados Unidos a decidir y ejecutar una invasión armada sobre aquel país).

- por otro lado, las perspectivas que vienen de más allá de las fronteras son útiles para iluminar las perspectivas propias de una comunidad determinada, pues permiten combatir contra lo que Sen denomina el provincianismo [parochialism].

Ese provincianismo que Sen atribuye a las teorías contractualistas tiene su equivalente jurídico en el dogma que identifica el Derecho con la producción local de normas jurídicas por parte de un Estado. Este dogma, que en su formulación clásica más extrema lleva a la identidad conceptual de Derecho y Estado (Kelsen dirá que ambas cosas literalmente son lo mismo ${ }^{8}$, controla los currículos y la pedagogía en las facultades de Derecho contemporáneas, hasta el punto de que se le ha considerado, siguiendo la terminología epistemológica de Thomas Khun, como el principio básico del paradigma dominante en la ciencia jurídica actual'

${ }^{8}$ H. Kelsen (1997) Teoría General del Derecho, México, Porrúa (traducción de Roberto J. Vernengo).

9 Entre otros, M. VAN DE KERCHOVE y F. Ost (1987) Jalons pour une théorie critique du droit, Bruselas, Facultés Universitaires Saint-Louis. 
El dogma del carácter nacional o estatal del Derecho es un importante factor a la hora de explicar la marginación de las cuestiones de justicia en las facultades jurídicas. Las razones aquí son las mismas que las que Sen utiliza para criticar el carácter excluyente de la teoría de la justicia rawlsiana. Por un lado las injusticias de nuestro mundo tienen alcance global y frente a ellas el Derecho nacional es irremediablemente estrecho, sea como perspectiva para contemplarlas y analizarlas, sea como intrumento para combatirlas. Por otro lado, la concentración en el Derecho nacional excluye perspectivas provenientes de más allá de las fronteras del Estado determinado del que se trate, convirtiendo así al jurídico en un ámbito condenado al provincianismo.

Sen entiende, en conformidad con la tradición ilustrada a la que no renuncia, que un elemento esencial de la justicia es la racionalidad. Ésta se traduce en que los juicios a partir de los cuales se toman decisiones y se actúa en el mundo no deben ser arbitrarios sino imparciales. Rawls apunta claramente hacia esa imparcialidad al tomar como base de su teoría lo que él llama la justicia como equidad [justice as fairness], que se manifiesta en el velo de ignorancia que permite a los individuos negociar en igualdad de condiciones en la posición original. Sin embargo, Sen califica esta imparcialidad rawlsiana como cerrada, en la medida en que está limitada a los miembros de una comunidad política concreta y no tiene en cuenta ni las consecuencias que ésta pueda tener en otras comunidades ni las perspectivas provenientes de esas otras comunidades que puedan iluminar y enriquecer a esta. Frente a ello Sen propone una imparcialidad abierta, en la que no sólo cuentan las perspectivas de aquellos que están cerca sino también las de aquellos que están lejos. La racionalidad no es una garantía de que nuestros juicios son necesariamente justos, pero al menos permite ser objetivos en la formulación de los mismos, y esa objetividad exige que dichos juicios puedan sobrevivir a un escrutinio informado, realizado desde ámbitos muy diferentes. En lugar de la posición original de Rawls, Sen toma aquí como referencia la metáfora del espectador imparcial [impartial spectator] del muy malinterpretado Adam Smith. Para ese espectador, igual que para las injusticias, los límites de los Estados son poco relevantes: como afirma Sen, puede que tengan relevancia estrictamente legal, pero no moral ${ }^{10}$.

${ }^{10}$ Sen utiliza el recurso del espectador imparcial no sólo para atacar el carácter excluyente y provinciano de la teoría contractualista, sino también su carácter institucionalista. A diferencia de la posición original de Rawls, a partir de la cual se pretende establecer unas instituciones justas, el espectador imparcial observa también los comportamientos reales de las personas y las realizaciones sociales. No sólo observa reglas e instituciones, sino también vidas humanas. 
Rawls fue consciente de los problemas de política internacional $y$, tras haber publicado la parte central de su teoría de la justicia, buscó una fundamentación política de los principios y normas del Derecho internacional en la última de sus publicaciones más importantes, The Laws of Peoples. En ella Rawls consideraba a los Estados como unidades independientes que se relacionan entre ellos de distintas maneras, incluida una hipotética posición original en la que participarían representantes de las distintas comunidades.

Sen concluye que dicho movimiento es insatisfactorio: internacional no es lo mismo que global, y la naturaleza de la justicia (o mejor, de las injusticias) es global y no (sólo) internacional. La teoría del Derecho de gentes de Rawls sigue manteniendo la centralidad de las comunidades políticas individualmente consideradas, de la misma manera que los principales sujetos del Derecho internacional siguen siendo los Estados que negocian y pactan entre ellos. El modelo que propone Sen tiene importantes limitaciones. Por un lado, esa concepción de lo internacional requiere de un apoyo institucional en la forma de un supraEstado global, cuya existencia, aun en caso de que fuera deseable, no parece inmediatamente plausible. Por otro lado, y esto es quizá más importante, tanto las víctimas como los agentes de las injusticias pueden ser individuos, grupos y organizaciones distintas a los Estados. Las interacciones entre todos esos agentes, que se producen con relativa independencia de las fronteras nacionales, no pueden reconducirse a una perspectiva de las relaciones internacionales basada en la concepción tradicional de relaciones entre Estados, que es la dominante en la teoría jurídica actual ${ }^{11}$.

Por todo ello, el dogma del carácter estatal del Derecho, que controla la pedagogía jurídica, no sólo es provinciano al excluir las perspectivas provenientes de otras partes del mundo, sino que es también reduccionista (incluso dentro de las fronteras nacionales) al no considerar la relevancia jurídica de agentes distintos al Estado, o al hacerlo sólo muy indirectamente. En este sentido es significativo que el estudio del fenómeno del pluralismo jurídico, que ataca el dogma del

\footnotetext{
${ }^{11}$ Estamos sosteniendo aquí que la teoría del Derecho internacional, en tanto en cuanto mantiene el dogma del carácter estatal del Derecho, contribuye a la marginación de las cuestiones de justicia en las facultades de Derecho. Pero merece la pena destacar que, incluso en esa concepción tradicional, el Derecho internacional está pobremente representado en el modelo de educación jurídica dominante. Así, por ejemplo, en el ámbito de la Unión Europea sorprende el escaso peso que en los currículos de los países miembros tiene el estudio de dicha institución, en contraste con la importancia que las normas y regulaciones europeas poseen en la vida jurídica de esos países. En la misma línea, pero en otro contexto regional, también llama la atención que en la mayoría de "Law Schools" de los Estados Unidos el Derecho internacional no sea una asignatura obligatoria.
} 
carácter estatal del Derecho en las dos dimensiones señaladas (por provinciano y por reduccionista), es un campo al que se le concede poca importancia en las facultades de Derecho, en contraste con su fertilidad en los ámbitos de la sociología y de la antropología social y cultural ${ }^{12}$.

\section{Misión de los jesuitas en relación con la educación jurídica}

La Congregación general $32^{a}$ formuló la misión de la Compañía de Jesús como "el servicio de la fe, del cual la promoción de la justicia es una exigencia absolu'a". En la Congregación general $34^{a}$, que desarrolló la comprensión de la misión de la Compañía en un contexto de postguerra fría y globalización, se afirmó que "el principio integrador de nuestra misión es el vínculo inseparable entre la fe y la promoción de la justicia en el Reino" (decreto 2, n. 14). De manera más específica, la Compañía realiza su misión de servicio de la fe dirigido a la promoción de la justicia a través de dos dimensiones: la atención a la cultura y la proclamación inculturada del Evangelio, así como el diálogo con otras tradiciones religiosas (Congregación general 34a , decreto 2, nn. 14-21). Ambas dimensiones están, a nuestro juicio, profundamente relacionadas con los puntos principales de la teoría de Sen que hemos analizado más arriba:

- Frente al carácter institucionalista, la Compañía toma como elemento esencial de la promoción de la justicia la "inculturación" o inserción en las culturas. En coherencia con la dualidad que propone Sen entre instituciones y realizaciones, la dimensión de la inculturación reconoce, junto a la importancia de las estructuras sociales y económicas formales (en las que las leyes e instituciones jurídicas tienen gran peso), la importancia no menos relevante de las actitudes culturales. La promoción de la justicia, igual que la proclamación del Evangelio, no debe hacerse "desde fuera" de la sociedad sino "desde dentro" de la misma. Así lo dice el decreto 3 de la Congregación general $34^{a}$, en el que se afirma que "esta justicia no puede realizarse si, al mismo tiempo, no se cuidan las dimensiones culturales de la vida social" (n. 18). En ese sentido señaló el

${ }^{12}$ Sobre la relevancia del pluralismo jurídico en esos ámbitos véase, por ejemplo, S. ENGLE MERRY (1988) "Legal Pluralism": Law and Society Review 22, p. 5 y en general el "reader" editado por P. SACK y J. ALECK (1992) Law and Anthropology, Dartmouth. Uno de los co-autores de esta reflexión analizó las implicaciones metodológicas del estudio del pluralismo jurídico y cómo estas chocan con el modelo de ciencia jurídica tradicional, C. ARJONA (2005) "Pluralismo vs. Dogmática: una aproximación epistemológica al fenómeno jurídico": Revista del Conesup, $\mathrm{n}^{\circ} 1$ 1, pp. 90-111 (inicialmente publicado en la serie Cuadernos de la Facultad de Derecho de ESADE, n 3, Barcelona, Universitat Ramon Llull, 2003). 
padre Kolvenbach, que: la promoción de la justicia significa una llamada a la Compañía para que nos insertemos cada vez más hondamente en la vida concreta de los pueblos y de las naciones (citado en Congregación general 34, decreto 3, n. 24).

- Frente al carácter excluyente, la Compañía considera que la otra dimensión esencial de la promoción de la justicia es el diálogo con otras tradiciones y religiones. Este diálogo está inspirado por la propia vida de Jesús, quien "supera las fronteras físicas y socio-religiosas" (Congregación general 35, decreto 3, n. 14). La Compañía representa en este ámbito un magnífico potencial, al ser una organización globalizada en sí misma, cuyos miembros y terrenos de acción abarcan una realidad abiertamente multicultural (Congregación general 35, decreto 3, n. 43). En la medida en que su tarea de promoción de la justicia se materialice en la pedagogía jurídica, los dogmas de carácter local, relacionados con la identificación entre Derecho y Estado, se antojan más un obstáculo que una buena fundación.

En definitiva, lo que Sen nos está diciendo, en un lenguaje no cristiano y no religioso, es que hay que promover la justicia luchando contra la injusticia, y que para hacerlo es necesario entrar en las culturas y es también necesario abrirse a, y dialogar con, otras tradiciones. $Y$ estos no son sino los elementos requeridos, según la propia misión de la Compañía de Jesús, para servir a la fe (Congregación general 34 , decreto 2 , n. 19).

\section{Conclusión}

Las maneras en que se puede materializar esa misión en una facultad de Derecho son múltiples. Apuntamos algunas líneas posibles:

- La insistencia en asignaturas de carácter no jurídico-dogmático, que se muevan en la dimensión que Sen denomina nyaya. Es esencial entender el contexto social en el que existe el Derecho y las consecuencias globales de su aplicación ${ }^{13}$.

\footnotetext{
${ }^{13}$ En este ámbito, en la Facultad de Derecho de ESADE, además de las asignaturas de Teoría y de Filosofía del Derecho, se imparten dos asignaturas propias de carácter obligatorio para todos los estudiantes: Filosofía Social y Ética Jurídica. También pueden contribuir decisivamente a introducir la cuestión de la justicia en una facultad asignaturas tales como la Sociología, la Antropología, la Política, la Argumentación, etc
} 
- En el aspecto curricular, la insistencia en las materias internacionales y comparativas, que sirvan no sólo para adquirir el conocimiento de normas de otros sistemas, sino sobre todo para abrir perspectivas que permitan entender y valorar mejor el propio sistema, y de esta manera luchar contra el provincianismo al que se refiere Sen.

- Desde un punto de vista antropológico, el trabajo en la formación integral del individuo como profesional y como persona. En esta dimensión deben situarse las actividades extra-curriculares que aportan experiencias vitales importantes para el proceso de formación entendido como proceso de cambio personal. ${ }^{14}$

En todas las anteriores líneas, la red formada por las instituciones jesuitas de educación superior constituye un muy valioso recurso para acometer la tarea. La Congregación general 35 nos habla de "la tradición de los jesuitas de tender puentes superando las fronteras" (decreto 3, n. 17); y precisamente lo que necesita la educación jurídica actual, a juicio de estos autores e interpretando la teoría de la justicia de Sen, es la construcción de puentes. Puentes entre las instituciones y la vida real, entre los libros y la acción, entre las leyes y las consecuencias de su aplicación. Puentes entre la facultad de Derecho y los otros ámbitos del conocimiento, en particular sociales y humanísticos, que pueden y deben iluminar a aquel sobre las realidades de la vida humana. Puentes, por supuesto, entre distintas tradiciones, sistemas de Derecho y culturas socio-jurídicas.

Las facultades de la Compañía de Jesús se hallan, pues, en una situación idónea para superar el carácter dogmático, formalista, local y excluyente que aún domina la educación jurídica. Lograrlo constituiría un gran servicio en la promoción de la justicia.

\footnotetext{
${ }^{14}$ Como ilustración, dentro de las actividades que se organizan en este ámbito en la Facultad de Derecho de ESADE, merece la pena destacar las "prácticas profesionales solidarias" en el marco del SUD (Servicio Universitario para el Desarrollo) que permiten a los alumnos realizar trabajo real durante un verano en países centroamericanos.
} 